\title{
Novel Methods to Harness Solar Radiation for Advanced Energy Applications
}

\author{
Yin Gao ${ }^{1}$, Ziman Wang ${ }^{1,2}$, Ding Ding ${ }^{3}$, Wenjia $\mathrm{Li}^{4}$, Yaoguang $\mathrm{Ma}^{5^{*}}$, Yong $\mathrm{Hao}^{1,2^{*}}$ and Hang Zhang ${ }^{1,2^{*}}$
}

With the demand for renewable energy, solar energy is widely used to generate and convert into heat. It is important to look beyond solar thermal for revolutionary technology to increase the coefficient of utilization of solar energy. In this editorial, a review on the promising novel technologies and applications for solar energy utilization is presented. In particular, we examine novel technologies for solar radiation controlling and harnessing solar energy for energy-efficient space heating and cooling through spectral selectivity and heat pump systems. These novel technologies in solar energy systems can provide significant benefits and can be developed for a wide range of applications.

Keywords: Solar radiation; Radiation regulation; Spectral selectivity; Heat pump

Accepted 29 September 2019

DOI: $10.30919 / \mathrm{esee} 8 \mathrm{c} 328$

\section{Introduction}

Lately, energy utilization and energy savings have become crucial concerns in the whole world. Oil, coal, and gas are the conventional energy sources we use and constitute almost $80 \%$ of global energy consumption. The equivalent of 60 billion boe of oil and gas which was about 8.2 billion tonnes of oil to run the world in 2018, ${ }^{1}$ and such a drastic consumption has a huge impact on our environment. ${ }^{2}$ Renewable and clean energy like solar, wind, biomass, hydropower and tidal energy bring us a competitive alternative if we can utilize them efficiently. ${ }^{3}$ Over the past five years, the demand for renewable energy is rapidly growing. The renewable energy are plying an important role in the world. Among the larger EU economies, the renewable energy share in power is $69 \%$ in Denmark, and $32 \%$ in Germany and UK, respectively. ${ }^{4}$ The coefficient of utilization for renewable energy is still poor despite the massive energy potential and the awareness of the advantages of the new energy. This is because renewable energy resources are generally not always reliable and accessible, giving rise to technical and economic challenges in utilizing these energy sources.

Among all the renewable energy sources, solar energy is the most promising option. It is free, clean and abundant and has attracted more attention from industries and researchers around the world. ${ }^{5}$ Innovative advances in technology and material science will increase the collection and conversion efficiencies. This will in turn further increase the reliability and applicability, and lower the costs of using solar energy

'Institute of Engineering Thermophysics, Chinese Academy of Sciences, Beijing 100190, China

${ }^{2}$ University of Chinese Academy of Sciences, Beijing 100190, China

${ }^{3}$ Institute of Materials Research and Engineering, A*STAR Research Entities 138632, Singapore

${ }^{4}$ Key Laboratory of Efficient Utilization of Low and Medium Grade Energy, MOE, Tianjin University, Tianjin 300350, China

5 State Key Laboratory of Modern Optical Instrumentation, College of Optical Science and Engineering, Zhejiang University, Hangzhou, Zhejiang 310013, China

*E-mail: mayaoguang@zju.edu.cn; haoyong@iet.cn; zhanghang@iet.cn and potentially solve our most critical energy problems.

To innovate better technologies, we often have to go back to the basic science. Solar energy is no exception. All thermodynamic objects emit radiation, with emissive power proportional to the fourth power of its temperature according to the Stefan-Boltzmann law. Matter exchanges energy with surroundings continuously by means of radiation, conduction and convection. The sun is essentially a heat source emtting electromannetic waves. Thus, spectral transmission, reflection and absorption take palce simultaneously when electromagnetic radiation impinges on a material, can be tailored to meet different demands in specific wavelength ranges. The heating and cooling for spaces purposes can be achieved by designing the radiative properties of the surfaces in different solar wavelength ranges.

There are a variety of novel technologies and applications for solar energy utilization. In this editorial, we discuss novel technologies for solar radiation controlling and solar thermal collector by the spectral selectivity and heat pump systems which are used for space heating and cooling.

\section{Technologies and applications for solar energy utilization}

2.1 Radiative heating materials

The radiative heating materials can be widely used in buildings ${ }^{6}$ and automobile windows ${ }^{7}$ for the energy-saving windows, textile for keeping warm, ${ }^{8}$ and aerospace to reduce the absorption of thermal radiation from the sun. ${ }^{9}$

Fig. 1 shows solar radiation and blackbody radiation at $360 \mathrm{~K}$, together with ideal spectral selective radiative heating and cooling materials. It can be observed that the solar radiation is limited between the $0.25 \mu \mathrm{m}$ and $3 \mu \mathrm{m}$ wavelength. The temperature-dependent blackbody radiation is peaked around $10 \mu \mathrm{m}$ wavelength when the temperature of the object is $360 \mathrm{~K}$ (Fig. 1). The controlled utilization of solar radiation is a key element in high-efficiency thermal management for both human and their buildings. ${ }^{8,10}$ In a cold climate, more solar energy is favorable while avoiding unwanted energy loss by thermal radiation. The ideal radiative heating materials should get as much 
sunlight as possible for the wavelength between $0.3 \mu \mathrm{m}$ and $3 \mu \mathrm{m}$ and lose as little as possible for the wavelength larger than $3 \mu \mathrm{m}$. However, in a warm climate, while maintaining the useful visible light, the rest of the solar energy (around 50\% of the total energy) should be blocked for cooling purposes. The ideal radiative cooling materials should have high transmittance for the wavelength between $0.4 \mu \mathrm{m}$ and $0.7 \mu \mathrm{m}$ and low transmittance for the rest of the wavelength. There is a significant atmospheric window occurring from $8 \mu \mathrm{m}$ to $13 \mu \mathrm{m}$ wavelength in which has the lowest atmospheric absorptance. It is possible to design passive cooling materials by increasing the spectral absorptance between the wavelength of $8 \mu \mathrm{m}$ and $13 \mu \mathrm{m}$. ${ }^{11}$ The radiative cooling materials are discussed in detail in section 2.3.

The spectral radiation of idealized radiative heating materials has been shown in Fig. 1. There are several types of radiative heating materials: 1) the surfaces with high solar absorptance and low thermal emittance could efficiently convert solar radiation into useful heat. ${ }^{13}$ For example, $\mathrm{Ni}$ pigmented anodized $\mathrm{Al}$ is one of the typical examples of spectral selective surfaces for efficient photothermal conversion of solar energy which was presented in $1980 .{ }^{14}$ Here the metal particles are much smaller than the wavelengths of the electromagnetic radiation. 2) the transparent in visible light and high infrared-reflecting coatings. On the one hand, the solar radiative heating materials work by reflecting the heat from the near-infrared solar energy. The typical material is doped conducting or semiconducting materials like indium tin oxide (ITO) and fluorine-doped tin oxide (FTO) with dopants such as Zn, In, and Sn. However, the dopant level will reach a limit during synthesis. On the other hand, the radiative heating materials exhibit high transmittance in the visible and near-infrared spectral region, and high reflectance and low emittance in the mid-infrared region as shown in Fig. 2. Thus, the sunlight can mostly enter indoor through the materials, and the infrared light inside is reflected back to achieve heating effect. It can be realized by metal (such as $\mathrm{Cu}, \mathrm{Ag}, \mathrm{Al}$ or $\mathrm{Au}$ ) thin films with the thickness smaller than wavelength. ${ }^{16}$ The so-called "low-E" glasses for buildings are based on metal films. With developing the nanotechnology, the metal nanowires were used for preparing the spectrally selective materials. Li et al. (DOI: 10.30919/esee8c325) successfully synthesized

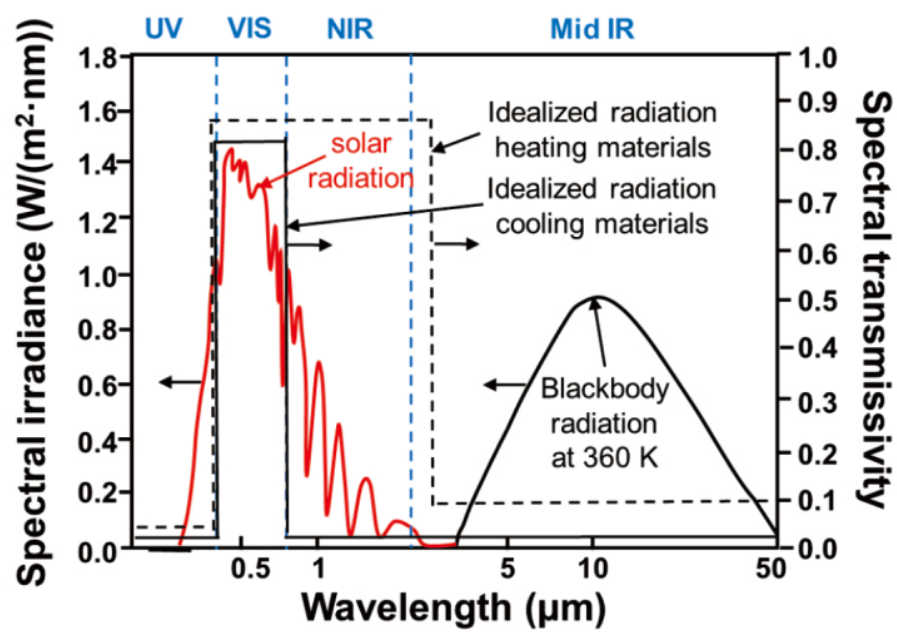

Fig. 1 Spectral distributions of solar radiation, blackbody radiation at $360 \mathrm{~K}$ and ideal spectral transmittance of radiation control materials which are marked by arrows. ${ }^{12}$

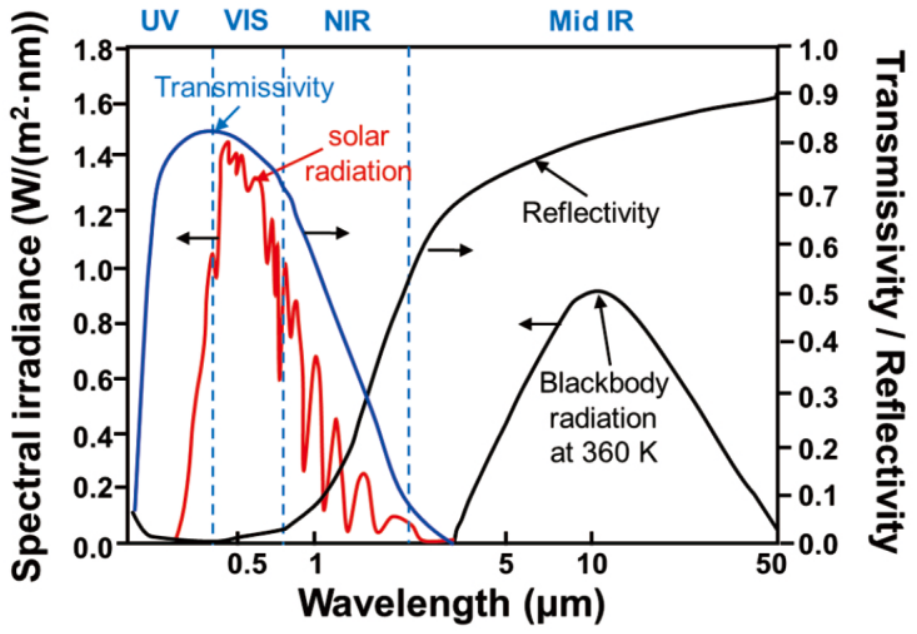

Fig. 2 Spectral transmission and reflection distributions of a kind of radiative heating materials with high transmittance in the visible and near-infrared spectral region and high reflectance in the mid-infrared region. 
metal-polymer hybrid metamaterials with high transmission through the visible wavelength range and high reflectance in the mid- and farinfrared ranges which is based on the Mie scattering and the surface plasmon enhanced transmission. The experimental results showed that their films warm up a prototype greenhouse 8 degrees Celsius higher than that of glasses on an average sunny day. Hsu et al. ${ }^{8}$ prepared metallic nanowire-embedded cloth with silver nanowires and carbon nanotubes by dip-coating which can efficiently warm human bodies and save hundreds of watts per person as compared to traditional indoor heaters.

\subsection{Solar thermal collector}

The solar thermal collector is one of the most efficient systems to convert the enormous amount of solar energy to usable energy such as heat or electricity. The efficiency depends on the spectral-selective solar absorbing materials.

In solar thermal applications, absorption of sunlight (i.e., input) and reemission of thermal radiation (i.e., loss) occur simultaneously. By the first law of thermodynamics and Planck's law of blackbody radiation, the absorption efficiency of blackbody solar absorber is given as, $^{17}$

$$
\eta_{a b s}=\frac{E_{\text {in }}-E_{\text {out }}}{E_{\text {in }}}=1-\frac{\sigma \cdot T^{4}}{I \cdot C}
$$

where $E_{\text {in }}$ is input solar power of the absorber, $E_{\text {out }}$ is power loss due to thermal radiation from the absorber, $\sigma$ is Stefan-Boltzmann constant, $T$ is the temperature of the absorber, $I$ is direct normal irradiance of sunlight, and $C$ is concentration ratio of the absorber. Take a typical absorber in a parabolic trough solar collector as an example, if the absorber was assumed to be a blackbody, about $17 \%$ of solar energy input would be lost via thermal radiation with a concentration ratio of 70 and an operating temperature of $400{ }^{\circ} \mathrm{C}$. For a non-blackbody absorber (e.g., a real parabolic trough solar collector), Eq. (1) is rewritten in a more general form, where for absorbers with selective coatings / micro-structure:

$$
\eta_{a b s}{ }^{\prime}=\alpha_{1}-\frac{\varepsilon_{1} \cdot \sigma \cdot T^{4}}{I \cdot C}
$$

while for absorbers with selective filters:

$$
\eta_{a b s}^{\prime}=\tau_{2}-\frac{\tau_{2}^{\prime} \cdot \sigma \cdot T^{4}+\varepsilon_{2} \cdot \sigma \cdot T^{\prime 4}}{I \cdot C}
$$

where $\alpha_{1}$ and $\varepsilon_{1}$ are absorptivity and emissivity of non-blackbody absorber with selective coatings or micro-structure, respectively; $\tau_{2}$ and $\tau_{2}^{\prime}$ are transmissivities of selective filter for solar spectrum and thermal radiation spectrum from non-blackbody absorber, respectively; $\varepsilon_{2}$ is emissivity of selective filter, and $T^{\prime}$ is temperature of selective filter. To reduce the radiative heat loss and improve the thermal efficiency, emissivity of a solar absorber must be suppressed while the absorptivity is kept as close as possible to that of a blackbody.

Selective coatings with high absorptivity of the incident solar spectrum (e.g., $300 \mathrm{~nm}-2500 \mathrm{~nm}$ ) and low emissivity of outlet thermal radiation spectrum (e.g., $2.5 \mu \mathrm{m}-25 \mu \mathrm{m}$ ) have been extensively studied. ${ }^{13}$ State-of-the-art absorptivity of a selective coating has been improved to higher than 0.95 , whereas emissivity has been reduced to lower than $0.05 .^{18,19}$ Correspondingly, the radiation loss of the absorber with the selective coating will decline to below $5 \%$ of that without the coating (Fig. 3 (a, c)).

In comparison, spectral selective filters are usually employed for solar cavity collectors to improve their thermal efficiency. The spectral regulation and control mechanism, however, are different from that of coatings, since cavity collectors themselves mimic blackbody receivers, and thus the transmission of such filters are tailored for designated wavelength ranges. Since the temperature of a solar cavity collector is significantly lower than that of the sun, a general design principle for such filters is that they shall be transmissive to major part of the solar spectrum and yet be reflective to the major part of the reemission spectrum (Fig. 3 (b, d)). The specific cutoff wavelength for such selectivity is determined by temperature requirement of the cavity receiver. Jin et al. ${ }^{20}$ increased the thermal efficiency of a solar methane reforming reactor (i.e., a cavity collector) from $71 \%$ to $76 \%$ by employing a spectral selective filter that transmits radiation with wavelengths below $2400 \mathrm{~nm}$ but blocks radiation with greater wavelengths from escaping through the window (Fig. 3 (d)), with (a)

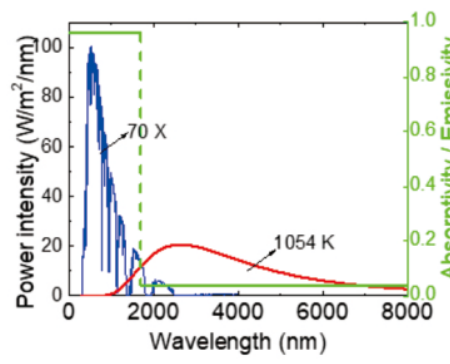

(c)

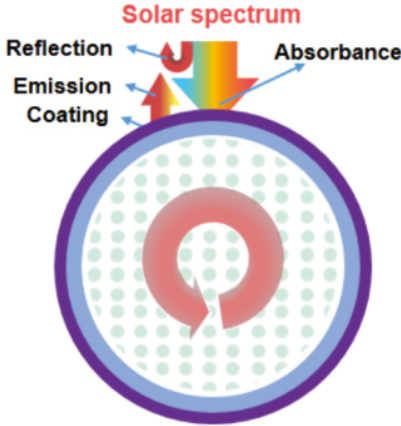

(b)

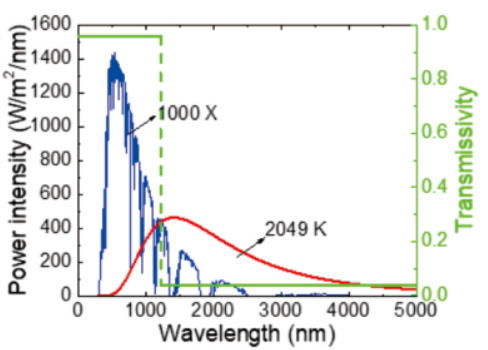

(d)

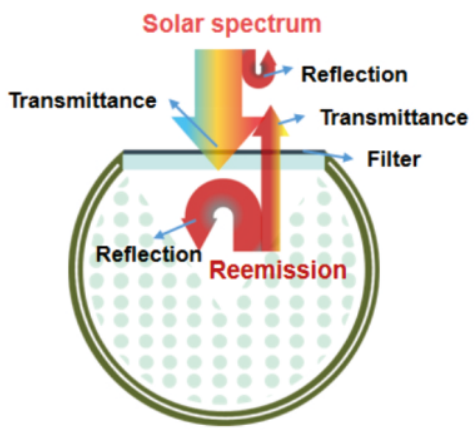

Fig. 3 Schematic diagram of solar thermochemical reactors and their spectrum assignments with spectral selective elements: (a) and (c) with selective coating; (b) and (d) with selective filter. 
concentration ratio up to 1000 and temperature up to $850{ }^{\circ} \mathrm{C}$. Similar effects by spectral selective filters are also reported for lowconcentration, low-temperature solar thermal collectors. By covering the reactor surface with spectral selective coating (Fig. 3 (c)), Zimmerman and Rosengarten ${ }^{21,22}$ successfully obtained operation temperature higher than $250{ }^{\circ} \mathrm{C}$ and carried out the reaction of hydrogen production via methanol-reforming without concentration or with very low concentration $(<2 \times)$.

\subsection{Radiative cooling materials}

Radiative cooling is a ubiquitous process that utilizes the huge temperature difference between the earth and the cold universe by emitting thermal radiations. Tremendous research has been conducted to systematically investigate the emissivity properties of radiative cooling surfaces, ${ }^{23,24}$ the environmental dependency of practical radiative cooling, ${ }^{25-28}$ new materials with desirable spectral properties ${ }^{11,29-31}$ and the applications of radiative cooling for buildings, ${ }^{32,33}$ solar panels, ${ }^{3436}$ water harvesting, ${ }^{37,38}$ outdoor personal thermal management, ${ }^{39}$ and supplemental cooling for power plants, ${ }^{40}$ etc.

The energy flow process between a given object and the environment can be simply described by the model shown in Fig. 4. The thermodynamic equilibrium equation for the system is:

$$
\frac{Q}{A} \frac{\partial T}{\partial t}=P_{n e t}
$$

where $Q$ is the heat capacity, $A$ is the area of the object. $T$ is the object's temperature.

The net radiative cooling power $\left(P_{n e}\right)$ of the object can be expressed as:

$$
P_{n e t}=P_{r a d}-P_{a t m}-P_{s u n}-P_{c o n}
$$

where $P_{\text {rad }}$ is the thermal radiation power from the object. $P_{a t m}$ is the power absorbed by the object from the atmosphere. $P_{s u n}$ is the absorbed solar irradiation power on the object. $P_{\text {con }}$ denotes the nonradiative heat transfer between the object and the environment. Here we neglect the effect of surroundings buildings, ground, etc. since in most cases radiative cooling surfaces are located horizontally in open space. The radiation power from the object can be obtained by integration of the spectrum in the sky hemisphere:

$$
P_{a t m}\left(T_{a t m}\right)=\int_{0}^{\pi / 2} \sin \theta \cos \theta d \theta \int_{0}^{\infty} I_{B B}\left(\lambda, T_{a m b}\right) \epsilon_{a t m}(\Omega, \lambda) d \lambda
$$

where $I_{B B}\left(T_{o b j}, \lambda\right)=\frac{2 h c^{2}}{\lambda^{5}} \frac{1}{e^{h c h k_{B} T_{b b /}}-1}$ is the blackbody radiation law.

The total absorbed atmospheric radiation power can be expressed as:

$P_{a t m}\left(T_{a t m}\right)=\int_{0}^{\pi / 2} \sin \theta \cos \theta d \theta \int_{0}^{\infty} I_{B B}\left(\lambda, T_{a m b}\right) \epsilon_{o b j}(\Omega, \lambda) \epsilon_{a t m}(\Omega, \lambda) d \lambda$

where, $\epsilon_{o b j}$ and $\epsilon_{a t m}$ are the emissivity of the object and the atmosphere respectively. $\epsilon_{\text {atm }}$ can be obtained from several computational tools such as MODTRAN, ${ }^{41}$ ATRAN, ${ }^{42}$ etc.

The total absorbed solar power can be expressed as

$$
P_{\text {sun }}=\int_{0}^{\infty} I_{\text {sun }}(\lambda) \epsilon_{o b j}\left(\lambda, \theta_{\text {sun }}\right) d \lambda
$$

where, $\theta_{\text {sun }}$ is the solar altitude angle, $I_{\text {sun }}$ is the spectrum of solar irradiances, such as AM1.5, etc. The absorptivity of the object equals to its emissivity according to Kirchhoff's law.

The nonradiative heat transfer power can be expressed as:

$$
P_{c o n}\left(T_{o b j}, T_{a t m}\right)=h\left(T_{a t m}-T_{o b j}\right)
$$

where $h$ is an overall heat transfer coefficient that accounts for both convection and conduction effects.

Although an object with close-to-unity emissivity has the largest cooling power, it also absorbs excess emission from the surroundings which probably have a higher temperature than the object. Thus, a cooling surface with spectral dependent emissivity always performs better. To optimize the emissivity in the atmospheric window, ideal materials would have molecular stretching and rotation modes and exhibit strong infrared absorption within the corresponding spectral range. For example, the $\mathrm{C}=\mathrm{C}$ bond has strong light absorption between $10 \mu \mathrm{m}$ and $12.5 \mu \mathrm{m}, \mathrm{C}-\mathrm{O}$ and $\mathrm{C}-\mathrm{N}$ stretching bonds are absorptive from $8 \mu \mathrm{m}$ to $10 \mu \mathrm{m}$, and deformation vibration of $\mathrm{OH}$ group provides IR absorption between $8 \mu \mathrm{m}$ and $10 \mu \mathrm{m}^{43}$

The materials often used for radiative cooling applications can be categorized into three types: polymeric materials, inorganic thin films, gases. All three kinds of materials are proved to be very effective with cooling power close to an ideal blackbody. But for daytime radiative cooling, the high demand on both solar reflectivity and MIR emissivity possessed stringent requirement on material selections. Attempts since the 1980s mainly utilized oxide thin films but failed to cool down under the sun. ${ }^{44}$

The developments of nanophotonic structures have made it possible to manipulate the optical properties of a radiative cooling surface at the subwavelength scale. In 2013, Rephaeli et al. ${ }^{45}$ predicted a

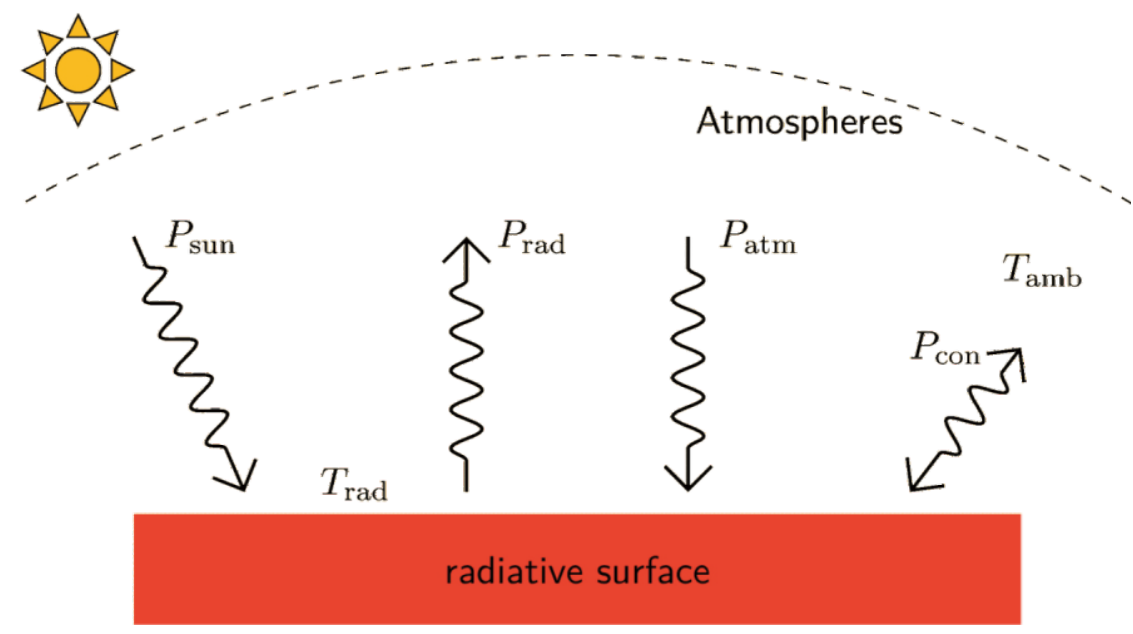

Fig. 4 Heat transfer processes on a radiative cooling surface. 
photonic crystal structure could achieve daytime radiative cooling. In 2014 , the same group reported ${ }^{31}$ sub-ambient daytime radiative cooling based on a simplified multilayer structure which consists of silicon dioxide and hafnium dioxide layers backed with a silver coating. Their cooler achieved a $40.1 \pm 4.1 \mathrm{~W} / \mathrm{m}^{2}$ cooling power and $4.9{ }^{\circ} \mathrm{C}$ temperature difference. Other designs based on similar metamaterial structures $^{46-50}$ have also been proposed and demonstrated high solar reflectivity and MIR emissivity.

In 2017, the Yin and Yang group ${ }^{11}$ at the University of Colorado reported a random metamaterial structure that is scalable and effective as depicted in Fig. 5. This extremely emissive metamaterial film consists of a TPX polymer matrix encapsulating randomly distributed silicon dioxide microspheres and a silverback coating layer (Fig. 5 (a)) and yields an average of $110 \mathrm{~W} / \mathrm{m}^{2}$ cooling power during continuous 3day measurement. Besides, the whole fabrication processes are scalable due to the mature roll-to-roll extrusion and coating techniques (Fig. 5 (b) and (c)). Later, Zhao et $a l^{40}$ utilized the random metamaterial film and built a $\mathrm{kW}$-scale radiative cooled cold collection and storage system. Other random-structure-based radiative cooling designs have also been reported since then. In 2018, Mandal et al. ${ }^{51}$ reported hierarchically porous polymer coatings for highly efficient passive daytime radiative cooling. The randomly generated porous structure exhibits hemispherical, $R_{\text {solar }}=0.96 \pm 0.03, \epsilon_{L W R}=0.97 \pm 0.02$ and a cooling power of $96 \mathrm{~W} / \mathrm{m}^{2}$. In 2019, Li et al. ${ }^{52}$ reported a radiative cooling material fabricated from conventional wood. By judiciously engineering wood with delignification and pressing, their cooling wood exhibit considerable radiative cooling ability.

Despite the appealing intrinsic "free" nature, limited cooling power density of radiative cooling technology restrains its applicable scenarios. One of the solutions is applying them in large scale cooling "farms" similar to the setup of the solar panel industry. For example, radiative cooling can be useful for large power plants, storages, off-grid area, and cruise ships since they have a large surface area with sky access. ${ }^{53}$ Cities can also benefit from daytime radiative cooling materials. Studies ${ }^{54}$ show that a $50 \%$ reflectivity difference between a regular roof and a high reflection roof yields a $20 \%$ energy use reduction. Zhao et al. ${ }^{55}$ conducted a filed test of shingle roof cooling of model-rooms and estimated that the energy saving can be as high as 91 $\mathrm{kWh} /\left(\mathrm{m}^{2} y r\right)$ for the analyzed location of Orlando, Florida, USA.

Harvesting water from air is another useful application of radiative cooling technology. Since the atmosphere contains 12900 cubic kilometers of available water, ${ }^{56}$ radiative-cooling based condensation provides a geographic-independent water collection tool for rural and other isolated locations. Several works reported by now can yield dew water with a speed of less than $0.2 l /\left(\mathrm{m}^{2}\right.$ day $) .^{5760}$ However, weathering conditions, such as clearness of the sky, relative humidity, and wind speed, etc. severely affects the output. More efforts are required to explore the nonlinear relationships between water production rate and climate conditions.

However, challenges still remain if we want to expand the horizon. The mismatch between the low cooling energy density $(\sim 100$ $\left.\mathrm{W} / \mathrm{m}^{2}\right)$ compared with the high cooling requirements for electronics $(\sim$ $\left.100 \mathrm{~W} / \mathrm{cm}^{2}\right)^{55}$ is still unsolved. Also, the configuration and efficiency for a systematic cooling apparatus are limited by the multilevel heat transfer processes. The tough management demand on both material side and system side place fetters for both scientists and engineers and requires more detailed researches.

\subsection{Solar-assisted heat pump systems}

Can we use solar energy to actively drive a refrigeration cycle to cool? Yes, we can. In fact, the simplest solution is to use a photovoltaic solar cell to drive a classical heat pump $^{61}$ or a thermoelectric cooler. ${ }^{62}$ Alternatively, one could use the heat from the sun to drive a thermal cycle and there is a wealth of reviews regarding solar-powered cycles for air-conditioning ${ }^{63,64}$ which will not be the focus of this editorial.

In this section, we will briefly postulate the possibility of a technology that uses sunlight to cool a material through an all-optical process-cooling via emission of upconverted photons. The idea of using upconversion for cooling has been explored using laser cooling of solids and has been successfully realized experimentally in crystals ${ }^{65-69}$ and semiconductors. ${ }^{70,71}$ In particular, cryogenic temperatures have been achieved in laser cooling ${ }^{66-68}$ of crystals and feasibly realized. ${ }^{65,72,73}$ The concept of upconversion cooling is illustrated in Fig. 6 (a). A photon absorbs heat energy from the thermal bath of the crystal by coupling to phonons and emitting an upconverted photon. Photon reabsorption and non-radiative processes severely limit the efficiency of the process and thus materials need to be specially selected with good purity ${ }^{67}$ and be of certain dimensions. ${ }^{74}$

Likewise, we can use near-field heat transfer in the form of thermal radiation to perform these upconversions. The process has been proposed recently, ${ }^{75,76}$ known as active thermal extraction, as shown in Fig. 6 (b), where instead of absorbing a phonon, the upconversion is made possible by coupling to a near-field photon. The energy of each photon can be larger than that of a phonon, making the process potentially more efficient. ${ }^{76}$ With an array of theoretical and experimental work demonstrating near-field thermal radiation control $^{77-80}$ and laser cooling of solids, ${ }^{81}$ the foundations for working towards such technology are certainly within reach. (a)

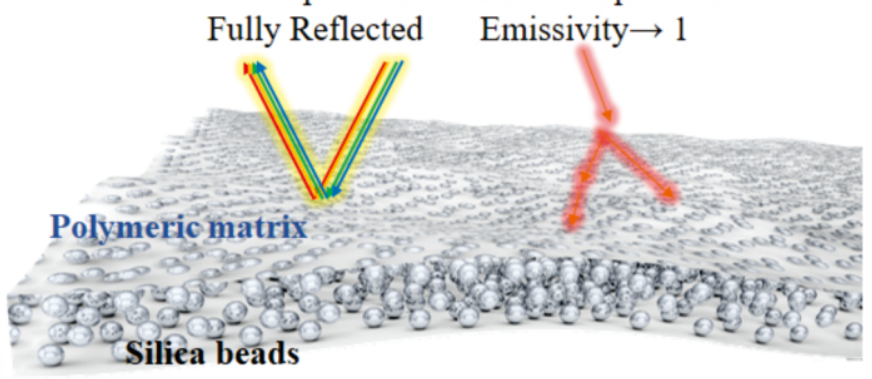

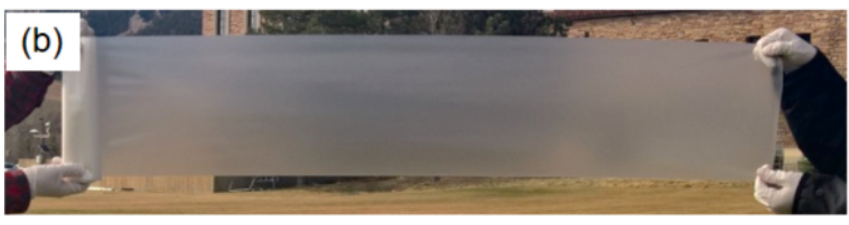

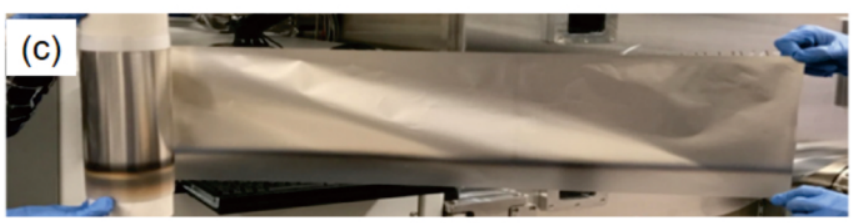

Fig. 5 Scalable-manufactured daytime radiative sky cooling metamaterial. ${ }^{11}$ (a) Schematic of the hybrid random metamaterial. (b) and (c) photos of the metamaterial film before and after the silver coating deposition. 
One may wonder if a coherent source such as a laser is absolutely necessary for these cooling methods. Actually, incoherent light of sufficient intensity will suffice. With optical concentration and spectral filtering, solar lasers have been realized decades back ${ }^{82,83,84}$ and similar techniques can be used for upconversion cooling using photons from the sun. One can envisage the availability of solar-assisted active cooling films like those passive cooling films discussed in section 2.3 in the future. Main challenges lie in using the right materials and systemlevel design to achieve high-efficiency cooling with sufficient sunlight intensity.

\section{Conclusions and outlook}

In this editorial, we discussed novel technologies, advantages and applications of solar radiation. We also examined how to improve solar energy utilization for industrial applications. Real-world applications of radiative heating or cooling technology remain a long-term challenge in terms of system integration. However, the combination of existing facilities and reinforced heating or cooling layers would enable continuously functioning systems and larger savings of electricity and water resources. Also, future developments of nanotechnology may also contribute to the manipulation of thermal properties of radiative controlling materials, and provide better tunability on the system performance. The potential for radiative controlling as an alternative technology coping with evolving environmental issues is certainly very promising. At the same time, active optical excitation to induce upconversion cooling are topics of great interest and using solar energy to achieve this would be extremely valuable in providing zero-energycost air-conditioners and refrigerators.

Methods discussed in this editorial could be combined. For example, a selective type solar collector-sky radiator which acts as a solar collector at daytime and as a sky radiator at night could be designed based on these spectral selective technologies.

Advanced materials and technologies still need to be developed. The configuration and efficiency for a systematic heating or cooling apparatus are limited by the multilevel heat transfer processes. The tough management demands both material and system advancements from both scientists and engineers in the field.

\section{Conflict of interest}

There are no conflicts to declare.

\section{Acknowledgments}

Y. Gao, Z. M. Wang, Y. Hao and H. Zhang acknowledge the financial support from the Basic Science Center Program for Ordered Energy Conversion of the National Natural Science Foundation of China (No.51888103) and the CAS Pioneer Hundred Talents Program. Y. G. Ma acknowledges the financial support from the Natural Science Foundation (No. 61905213). D. Ding acknowledges the support from Agency for Science, Technology and Research under Proposal (No. 17284017). W. J. Li and Y. Hao acknowledge the financial support from Chinese Academy of Sciences International Collaboration Key Program (182211KYSB20160043).

\section{References}

1. Conventional oil and gas discoveries show upstick in 2018, https://www.diesel net.com/news/2018/12oil.php, 2018.

2. S. A. Kalogirou, Prog. Energy Combust. Sci., 2004, 30, 231-295.

3. H. Schnitzer, C. Brunner and G. Gwehenberger, J. Clean. Prod., 2007, 15, 1271-1286.

4. Statistical Review of World Energy, https://www.bp.com/en/global/corporate/ energy-economics/statistical-review-of-world-energy.html, 2018.

5. S. Mekhilef, R. Saidur and A. Safari, Renew. Sustain. Energy Rev., 2011, 15, 1777-1790.

6. E. Hammarberg and A. Roos, Thin Solid Films, 2003, 442, 222-226.

7. E. Eby, R. O'Shaughnessy, R. Bond, High Transmittance, Low Emissivity Coating for Substrates 1994, 5302449.

8. P. C. Hsu, X. Liu, C. Liu, X. Xie, H. R. Lee, A. J. Welch, T. Zhao and Y. Cui, Nano Lett., 2015, 15, 365-371.

9. K. Sun, W. Zhou, X. Tang and F. Luo, Infrared Phys. Technol., 2016, 78, 156-161.

10. M. Casini, Int. J. Civ. Struct. Eng. - IJCSE, 2015, 273-281.

11. Y. Zhai, Y. Ma, S. N. David, D. Zhao, R. Lou, G. Tan, R. Yang and X. Yin, (a)

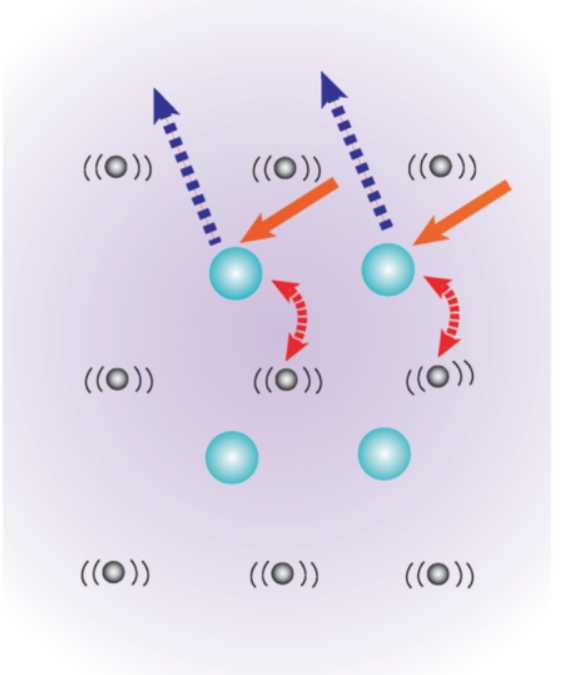

(b)

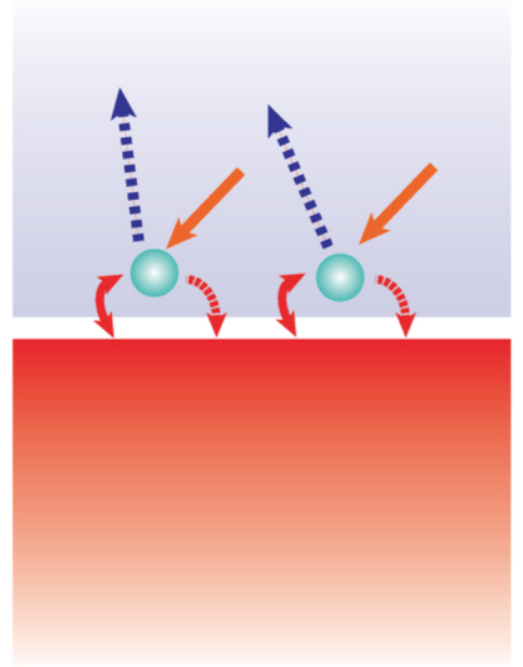

Fig. 6 (a) Schematic of the concept in laser cooling of solids. The laser medium consists of rare-earth emitters doped in a host crystal at some finite temperature. A light source excites the rare-earth emitter, and by absorption of a phonon, carry the energy away as upconverted emission. (b) Schematic showing the concept of active thermal extraction. A rare earth-doped medium similar to those used in laser cooling of solids is placed in the near-field of a substrate. The external light source excites the rare-earth emitter and results in upconverted emission due to coupling to the near-field thermal radiation from the substrate. ${ }^{76}$ 
Science, 2017, 355, 1062-1066.

12. C. G. Granqvist, Appl. Opt., 1981, 20, 2606-2615.

13. G. K. Manikandan, S. Iniyan and R. Goic, Appl. Energy, 2019, 235, 1524-1540.

14. Å. Andersson, O. Hunderi and C. G. Granqvist, J. Appl. Phys., 1980, 51, 754.

15. C. G. Granqvist, Sol. Energy Mater. Sol. Cells, 2007, 91, 1529-1598.

16. E. Valkonen, B. Karlsson and C. G. Ribbing, Sol. Energy, 1984, 32, 211-222.

17. E. A. Fletcher and R. L. Moen, Science, 1977, 197, 1050-1056.

18. M. Zettl, Energy Procedia, 2014, 48, 701-706.

19. A. Dan, H. C. Barshilia, K. Chattopadhyay and B. Basu, Renew. Sustain. Energy Rev., 2017, 79, 1050-1077.

20. J. Jin, X. Wei, M. Liu, Y. Yu, W. Li, H. Kong and Y. Hao, Appl. Energy, 2018, 226, 797-807.

21. R. Zimmerman, G. Morrison and G. Rosengarten, J. Sol. Energy Eng. Trans. ASME, 2010, 132, 011005

22. X. Gu, R. A. Taylor, G. Morrison and G. Rosengarten, Appl. Energy, 2014, $119,467-475$

23. C. G. Granqvist and A. Hjortsberg, Appl. Phys. Lett., 1980, 36, 139-141.

24. Berdahl, Appl. Opt., 1984, 23, 370.

25. C. D. Reid and E. D. McAlister, JOSA, 1959, 49, 78-82.

26. M. Hori, T. Aoki, T. Tanikawa, H. Motoyoshi, A. Hachikubo, K. Sugiura, T. J. Yasunari, H. Eide, R. Storvold, Y. Nakajima and F. Takahashi, Remote Sens. Environ., 2006, 100, 486-502.

27. A. P. Tsoy, A. S. Granovskiy, A. V Baranenko and D. A. Tsoy, AIP Conf. Proc. 2017, 1876, 020060.

28. S. Zhang and J. Niu, Energy Build., 2012, 54, 122-130.

29. R. Family and M. P. Menguc, Procedia Environ. Sci. 2017, 38, 752-759.

30. C. G. Granqvist, in KirkOthmer Encyclopedia of Chemical Technology, John Wiley \& Sons, Inc., 2017.

31. A. P. Raman, M. A. Anoma, L. Zhu, E. Rephaeli and S. Fan, Nature, 2014, $515,540-544$.

32. X. Lu, P. Xu, H. Wang, T. Yang and J. Hou, Renew. Sustain. Energy Rev. 2016, 65, 1079-1097.

33. U. Eicker and A. Dalibard, Sol. Energy, 2011, 85, 1322-1335.

34. L. Zhu, A. P. Raman and S. Fan, Proc. Natl. Acad. Sci., 2015, 112, $12282-12287$.

35. W. Li, Y. Shi, K. Chen, L. Zhu and S. Fan, ACS Photonics, 2017, 4, $774-782$

36. D. Du, J. Darkwa and G. Kokogiannakis, Sol. Energy, 2013, 97, 238-254.

37. J. F. Maestre-Valero, R. Ragab, V. Martinez-Alvarez and A. Bailie, $J$. Hydrol., 2012, 460, 103-109.

38. D. Carvajal, J. G. Minonzio, E. Casanga, J. Munoz, A. Aracena, S. Montecinos and D. Beysens, J. Water Supply Res. Technol., 2018, 67, 357-374.

39. L. Cai, A. Y. Song, W. Li, P. C. Hsu, D. Lin, P. B. Catrysse, Y. Liu, Y. Peng, J. Chen, H. Wang, J. Xu, A. Yang, S. Fan and Y. Cui, Adv. Mater, 2018, 30, 1802152.

40. D. Zhao, A. Aili, Y. Zhai, J. Lu and R. Yang, Joule, 2019, 3, 111-123.

41. MODTRANV R, http//modtran.spectral.com/Introd. about MODTRAN.

42. S. A. Lord, https//atran.sofia.usra.edu/cgi-bin/atran/atran.cgi Introd. about ATRAN.

43. T. S. Eriksson, E. M. Lushiku and C. G. Granqvist, Sol. Energy Mater, 1984, 11, 149-161.

44. G. B. Smith and C. G. S. Granqvist, Green Nanotechnology: Solutions for Sustainability and Energy in the Built Environment, CRC Press, 2010

45. E. Rephaeli, A. Raman and S. Fan, Nano Lett., 2013, 13, 1457-1461.

46. M. M. Hossain, B. Jia and M. Gu, Adv. Opt. Mater., 2015, 3, 1047-1051.

47. C. Zou, G. Ren, M. M. Hossain, S. Nirantar, W. Withayachumnankul, T. Ahmed, M. Bhaskaran, S. Sriram, M. Gu and C. Fumeaux, Adv. Opt. Mater. $2017, \mathbf{5}, 1700460$.

48. T. Liu and J. Takahara, Opt. Express, 2017, 25, A612-A627.

49. Wu, C. Liu, Z. Xu, Y. Liu, Z. Yu, L. Yu, L. Chen, R. Li, R. Ma and H. Ye,
Mater. Des., 2018, 139, 104-111.

50. Z. F. Huang and X. Ruan, Int. J. Heat Mass Transf., 2017, 104, 890-896.

51. J. Mandal, Y. Fu, A. Overvig, M. Jia, K. Sun, N. Shi, H. Zhou, X. Xiao, N. Yu and Y. Yang, Science, 2018, 362, 315-319.

52. T. Li, Y. Zhai, S. He, W. Gan, Z. Wei, M. Heidarinejad, D. Dalgo, R. Mi, X Zhao, J. Song, J. Dai, C. Chen, A. Aili, A. Vellore, A. Martini, R. Yang, J. Srebric, X. Yin and L. Hu, Science, 2019, 364, 760-763.

53. E. Mu, Z. Wu, Z. Wu, X. Chen, Y. Liu, X. Fu and Z. Hu, Nano Energy, 2019, 55, 494-500.

54. H. Akbari, P. Berdahl, R. Levinson and S. Wiel, Cool Color Roofing Materials, Lawrence Berkeley National Laboratory, 2006.

55. D. Zhao, A. Aili, Y. Zhai, S. Xu, G. Tan, X. Yin and R. Yang, Appl. Phys. Rev., 2019, 6, 021306.

56. Inner Earth May Hold More Water Than the Seas, https://news.nationalgeographic. com/news/2002/03/0307 0307 waterworld.html for information on water in the atmosphere, 2017.

57. A. F. G. Jacobs, B. G. Heusinkveld and S. M. Berkowicz, Atmos. Res., 2008 , 7, 377-385

58. M. Muselli, D. Beysens, M. Mileta and I. Milimouk, Atmos. Res., 2009, 92, 455-463.

59. P. Gandhidasan and H. I. Abualhamayel, Desalination, 2005, 180, 47-51.

60. G. Sharan, D. Beysens and I. Milimouk-Melnytchouk, J. Arid Environ., 2007, 69, 259-269.

61. Y. Li and R. Z. Wang, In: Wang RZ, Ge TS (eds). Advances in Solar Heating and Cooling. Woodhead Publishing, 2016, 227-250.

62. Y. L. Chen, Z. J. Chien, W. S. Lee, C. S. Jwo and K. C. Cho, Int. J. Photoenergy, 2014.

63. A. Al-Alili, Y. Hwang and R. Radermacher, Int. J. Refrig., 2014, 39, 4-22.

64. D. S. Kim and C. A. Infante Ferreira, Int. J. Refrig., 2008, 31, 3-15.

65. B. C. Edwards, J. E. Anderson, R. I. Epstein, G. L. Mills and A. J. Mord, J Appl. Phys., 1999, 86, 6489-6493.

66. R. I. Epstein, 3rd Annual Workshop on Laser Cooling of Solids, 2007.

67. G. Nemova and R. Kashyap, Reports Prog. Phys., 2010, 73, 86501.

68. D. V Seletskiy, S. D. Melgaard, S. Bigotta, A. Di Lieto, M. Tonelli and M. Sheik-Bahae, Nat. Photonics, 2010, 4, 161-164.

69. M. Sheik-Bahae and R. I. Epstein, Nat. Photonics, 2007, 1, 693-699.

70. S. T. Ha, C. Shen, J. Zhang and Q. Xiong, Nat. Photonics, 2016, 10, $115-121$.

71. J. Zhang, D. Li, R. Chen and Q. Xiong, Nature, 2013, 493, 504-508.

72. B. Zhong, H. Luo, Y. Shi and J. Yin, Opt. Eng., 2016, 56, 11102.

73. P. B. Roder, B. E. Smith, X. Zhou, M. J. Crane and P. J. Pauzauskie, Proc. Natl. Acad. Sci., 2015, 201510418

74. D. Li, J. Zhang and Q. Xiong, Opt. Express, 2013, 21, 19302-19310.

75. D. Ding, T. Kim and A. J. Minnich, Phys. Rev. B, 2016, 93, 81402.

76. D. Ding, T. Kim and A. J. Minnich, Sci. Rep., 2016, 6, 32744.

77. S. V. Boriskina, J. K. Tong, W. C. Hsu, B. Liao, Y. Huang, V. Chiloyan and G. Chen, Nanophotonics, 2016, 5, 1-27.

78. K. Kim, B. Song, V. Fernández-Hurtado, W. Lee, W. Jeong, L. Cui, D. Thompson, J. Feist, M. T. H. Reid, F. J. García-Vidal, J. C. Cuevas, E. Meyhofer and P. Reddy, Nature, 2015, 528, 387-391.

79. S. Molesky and Z. Jacob, Phys. Rev. B, 2015, 91, 205435

80. S. Shen, A. Narayanaswamy and G. Chen, Nano Lett., 2009, 9, 2909-2913.

81. J. B. Khurgin, Phys. Rev. Lett., 2007, 98, 177401

82. P. Gleckman, Appl. Opt., 1988, 27, 4385-4391.

83. A. L. Golger and I. I. Klimovskiī, Sov. J. Quantum Electron., 1984, 14, 164-178.

84. T. Ohkubo, T. Yabe, K. Yoshida, S. Uchida, T. Funatsu, B. Bagheri, T. Oishi, K. Daito, M. Ishioka, Y. Nakayama, N. Yasunaga, K. Kido, Y. Sato, C. Baasandash, K. Kato, T. Yanagitani and Y. Okamoto, Opt. Lett., 2009, 34, 175-177.

Publisher's Note Engineered Science Publisher remains neutral with regard to jurisdictional claims in published maps and institutional affiliations. 